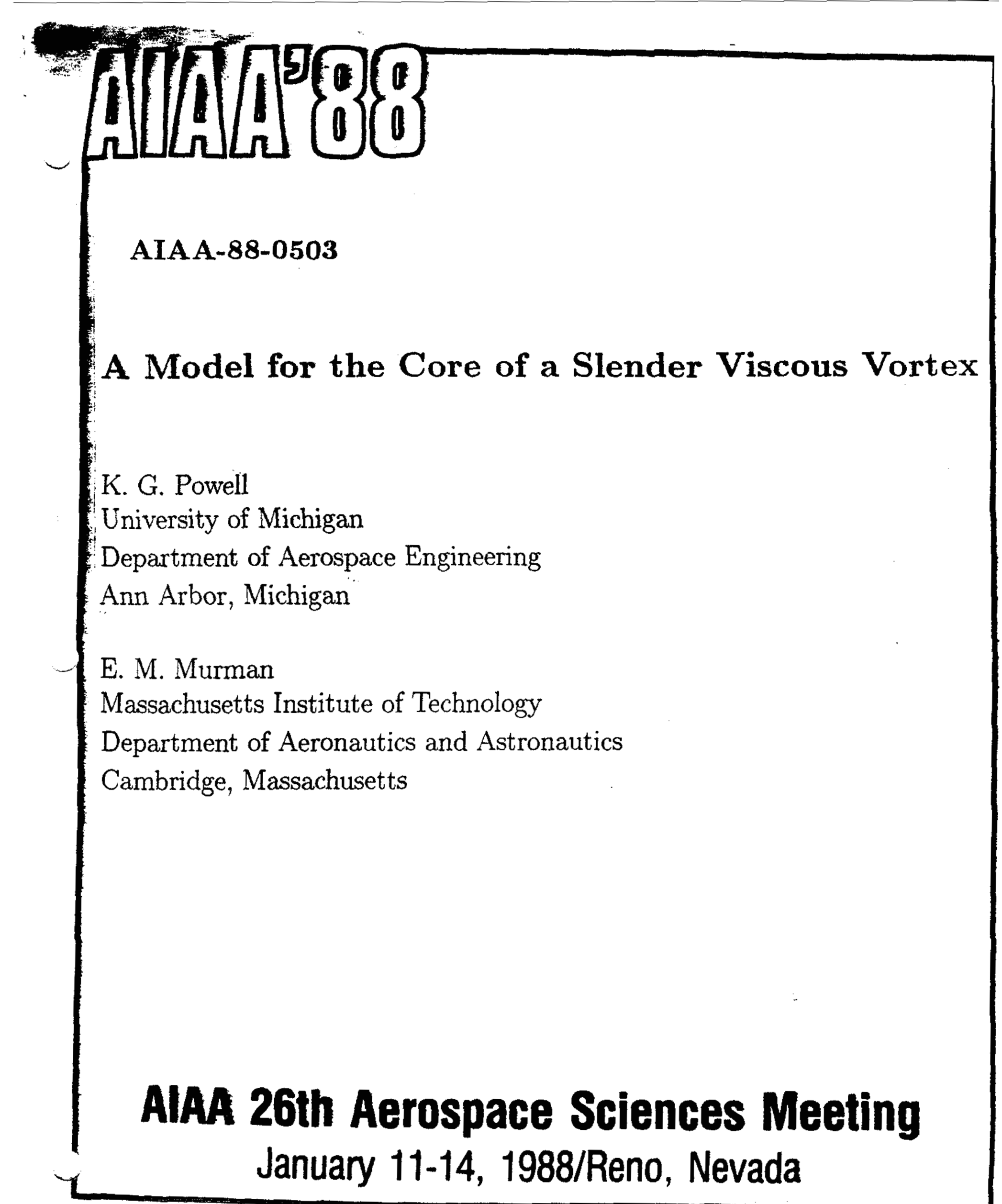

For permisaton to copy or republish, contact the Amorican Institute of Aoronauties and Astronautles 370 L'Enfant Promenade, S.W., Washington, D.C. 20024 


\section{A Model for the Core of a Slender Viscous Vortex}

\author{
Kenneth G. Powell* \\ The University of Michigan \\ Department of Aerospace Engineering
}

\author{
Earll M. Murman ${ }^{\dagger}$ \\ Massachusetts Institute of Technology \\ Department of Aeronautics and Astronautics
}

\begin{abstract}
- the flow is incompressible;

- the flow is axisymmetric;

- the flow is conical;

- the Reynolds number is high;

- the vortex is slender.
\end{abstract}

A model for the core of a viscous vortex is presented. It is based on a similarity solution to the Navier-Stokes equations in which the following assumptions are made:

Under these assumptions, the Navier-Stokes equations and the continuity equation take the form of four coupled, nonlinear, ordinary differential equations. These equations are discretized using centered differences, and solved by a Newton procedure, after imposing appropriate boundary conditions. The model is found to agree fairly well with experimental data of Earnshaw [1], and very well with the matched asymptotic solution for a vortex core developed by $\mathrm{Hall}$ [2]. The level of total pressure loss predicted in the core of the vortex is found to be independent of Reynolds number, depending only on the edge circumferential velocity. Some implications of this result on the prediction of the onset of vortex bursting are given.

\section{Introduction}

Due to the critical role that vortices play in aeronautical engineering, naval engineering and meteorology, a number of models for vortex cores have been developed through the years. These have ranged from very simple ad hoc models to complicated multiple-scale analyses. In this paper, the vortex core will be modeled as a region of distributed vorticity. Similar models have been proposed by Long [3], Hall [2], Luckring [4] and Brown [5], among others.

While a vortex core of distributed vorticity can occur only in a viscous flow, Euler calculations of vortex flows by the authors $[6,7]$ and others $[8,9,10]$ indicate that the discrete Euler equations model the flow inside a vortex core surprisingly well. In particular, the level of total pressure loss inside the core of a given vortex computed by the discrete Euler method is independent of computational parameters such as mesh spacing and artificial viscosity level [11], and agrees well with losses measured in experiment

\footnotetext{
- Assistant Professor, AlAA Member

${ }^{\dagger}$ Professor, AIAA Fellow
}

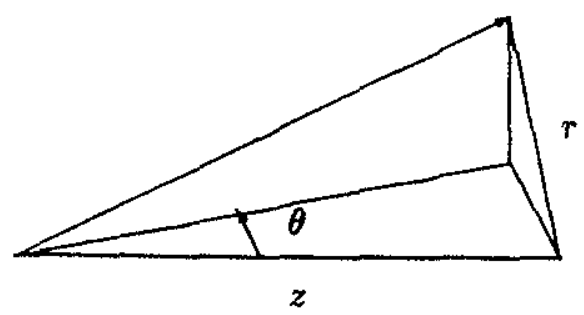

Figure 1: Cylindrical Coordinate System

[12]. Navier-Stokes calculations $[9,13,14]$ have comparable losses. This numerical evidence has driven the authors to determine whother there is a vortex core model which accounts for viscosity, but has a total pressure loss level that is independent of the level of viscosity.

\section{Burgers' Vortex}

Burgers developed a core model [15] that, it will be shown, has this characteristic. It is an exact solution to the axisymmetric, incompressible Navier-Stokes equations. For the cylindrical $(r, \theta, z)$ system, shown in Figure 1, the velocity field is given by

$$
\begin{aligned}
& u=-A r \\
& v=v(r) \\
& w=2 A z
\end{aligned}
$$

where $v(r)$ is to be determined from the $\theta$-momentum equation. The velocities are non-dimensionalized by a reference velocity $W$, and $r$ and $z$ are non-dimensionalized by a reference length $L$. This velocity field satisfies the continuity equation,

$$
\frac{1}{r} \frac{\partial}{\partial r}(r u)+\frac{\partial w}{\partial z}=0
$$

identically. The r-momentum equation (with pressure nondimensionalized by $\rho W^{2}$ ) reduces, for the above choices of $u, v$ and $w$, to

$$
\frac{\partial p}{\partial r}=\frac{v^{2}}{r}-u \frac{\partial u}{\partial r}
$$

The $z$-momenturn equation reduces to

$$
\frac{\partial p}{\partial z}=-w \frac{\partial w}{\partial z}
$$


and the $\theta$-momentum equation becomes

$$
u \frac{\partial v}{\partial r}+\frac{u v}{r}=\frac{1}{R e}\left(\frac{\partial^{2} v}{\partial r^{2}}+\frac{1}{r} \frac{\partial v}{\partial r}-\frac{v}{r^{2}}\right) .
$$

Burgers' original analysis was carried out in unscaled variables. The relation between the total pressure and the Reynolds number may be seen more easily, however, by rescaling the equations. Introducing scaled variables

$$
\begin{aligned}
& \hat{u}=u \sqrt{\frac{A R e}{2}} \\
& \hat{v}=\frac{A v}{2} \\
& \hat{w}=w \\
& \hat{p}=p \\
& \hat{r}=r \sqrt{\frac{A R e}{2}} \\
& \hat{z}=z
\end{aligned}
$$

does not affect the continuity equation. The $\theta$-momentum equation becomes

$$
\frac{\partial^{2} \hat{v}}{\partial \hat{r}^{2}}+\left(\frac{1}{\hat{r}}+2 \hat{r}\right) \frac{\partial \hat{v}}{\partial \hat{r}}+\left(2-\frac{1}{\hat{r}^{2}}\right) \hat{v}=0 .
$$

Applying the boundary conditions of zero swirl on the vortex axis and a potential flow at large radius,

$$
\begin{array}{ccc}
\hat{v}=0 & \text { at } \quad \hat{r}=0 \\
\hat{r} \hat{v} \rightarrow A \hat{\Gamma} / 2 & \text { as } \quad \hat{r} \rightarrow \infty
\end{array}
$$

yields the solntion

$$
\hat{v}=\frac{A \hat{\Gamma}}{2 \hat{r}}\left(1-e^{-t^{2}}\right)
$$

where $\hat{\Gamma}$ is a scaled circulation, related to the physical circulation $\Gamma$ by

$$
\hat{\Gamma}=\frac{\Gamma}{2 \pi} \sqrt{\frac{A R e}{2}}
$$

The $r$ and $z$ momentum equations, when integrated, yield the relation for the static pressure:

$$
\hat{p}=\frac{4}{A^{2}} \int \frac{\hat{v}^{2}}{\hat{r}} d \hat{v}-\frac{A \hat{r}^{2}}{R e}-2 A^{2} \hat{z}^{2} .
$$

From this the total pressure may be calculated, giving

$$
\begin{aligned}
p_{0} & =\frac{4}{A^{2}}\left[\int \frac{\hat{v}^{2}}{\hat{r}} d \hat{r}+\frac{\hat{v}^{2}}{2}\right] \\
& =\hat{\Gamma}^{2}\left[\int\left(\frac{1-e^{-t^{2}}}{\hat{r}}\right)^{2} \frac{d \hat{r}}{\hat{r}}+\frac{1}{2}\left(\frac{1-e^{-\hat{r}^{2}}}{\hat{r}}\right)^{2}\right] .
\end{aligned}
$$

Figure 2 is a plot of $p_{0} / \hat{\Gamma}^{2}$ as a function of $\hat{r}$ which holds for any Reynolds number. This means that the level of the total pressure loss in the core is set solely by $\hat{\Gamma}$, where

$$
\hat{\Gamma} \sim \Gamma \sqrt{A R e} .
$$

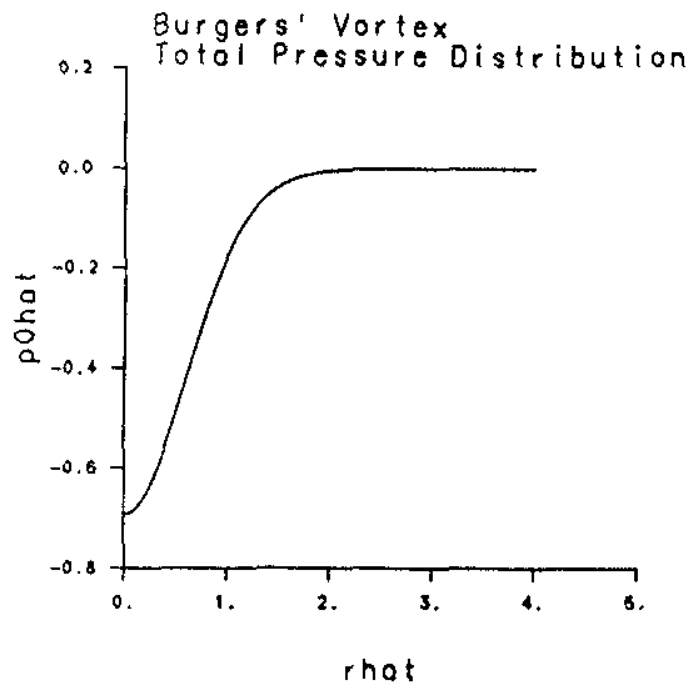

Figure 2: Burgers' vortex $-p_{0} / f^{2}$ vs $\hat{r}$

The position of the "edge" of the vortex, $r_{e}$, scales with $1 / \sqrt{A R e}$, which implies that

$$
\Gamma \sim v_{a} r_{a} \sim \frac{v_{e}}{\sqrt{A R e}}
$$

Thus,

$$
\hat{\Gamma} \sim v_{e}
$$

and, for a given edge circumferential velocity, the total pressure loss level in a Burgers' vortex is independent of Reynolds number. If the value of the edge velocity, $v_{e}$, is known, the magnitude of the total pressure loss in the core is determined. This is not an obvious result - the static pressure depends on the Reynolds number, as does the radial velocity. The distribution of the total pressure loss does depend on the Reynolds number; it scales with $1 / \sqrt{R e}$.

\section{Conical Core Model}

While Burgers' vortex exhibits the desired independence of Reynolds number for the level of the total pressure loss, the velocity field is necessarily a very contrived one. In this section of the paper, a conical analog to Burgers' vortex is derived which exhibits the same behavior for the total pressure. The assumption is made that, in the $\theta$-momentum equation, the viscous terms are not negligible, but of the same order as the convective terms. The core is also assumed to be slender and conical. While a viscous flow may not be truly conical, the approximation is a reasonable one due to the high Reynolds number.

The equations of motion for a steady, axisymmetric, viscous incompressible flow are the continuity equation,

$$
\frac{1}{r} \frac{\partial}{\partial r}(r u)+\frac{\partial w}{\partial z}=0
$$

the r-momentum equation,

$u \frac{\partial u}{\partial r}+w \frac{\partial u}{\partial z}-\frac{v^{2}}{r}=-\frac{\partial p}{\partial r}+\frac{1}{R e}\left[\frac{1}{r} \frac{\partial}{\partial r}\left(r \frac{\partial u}{\partial r}\right)+\frac{\partial^{2} u}{\partial z^{2}}-\frac{u}{r^{2}}\right]$ 
the $\theta$-momentum equation,

$$
u \frac{\partial v}{\partial r}+w \frac{\partial v}{\partial z}+\frac{u v}{r}=\frac{1}{R e}\left[\frac{1}{r} \frac{\partial}{\partial r}\left(r \frac{\partial v}{\partial r}\right)+\frac{\partial^{2} v}{\partial z^{2}}-\frac{v}{r^{2}}\right],
$$

and the $z$-momentum equation,

$$
u \frac{\partial w}{\partial r}+w \frac{\partial w}{\partial z}=-\frac{\partial p}{\partial z}+\frac{1}{R e}\left[\frac{1}{r} \frac{\partial}{\partial r}\left(r \frac{\partial w}{\partial r}\right)+\frac{\partial^{2} w}{\partial z^{2}}\right]
$$

where the Reynolds number is based on a reference length $L$ and a reference velocity $W$. Introducing the conical variables $\phi=r / z$ and $\zeta=\sqrt{r^{2}+z^{2}}$ and assuming conical self-similarity (that the solution is independent of $\zeta$ ), the continuity equation becomes

$$
-\phi\left(w-\frac{u}{\phi}\right)+2 \frac{u}{\phi}=0 .
$$

The r-momentum equation becomes

$$
\begin{aligned}
-\phi\left(w-\frac{u}{\phi}\right) u^{\prime}-\frac{v^{2}}{\phi} & =-p^{\prime}+ \\
\frac{1}{R e_{x}}\left[\left(1+\phi^{2}\right) u^{\prime \prime}+\left(\frac{1}{\phi}+2 \phi\right) u^{\prime}-\frac{u}{\phi^{2}}\right], & ,
\end{aligned}
$$

the $\theta$-momentum equation becomes

$$
\begin{aligned}
&-\phi\left(w-\frac{u}{\phi}\right) v^{\prime}+\frac{u v}{\phi}= \\
& \frac{1}{\operatorname{Res}}\left[\left(1+\phi^{2}\right) v^{\prime \prime}+\left(\frac{1}{\phi}+2 \phi\right) v^{\prime}-\frac{v}{\phi^{2}}\right]
\end{aligned}
$$

and the $z$-momentum equation is given by

$$
\begin{aligned}
-\phi\left(w-\frac{u}{\phi}\right) w^{\prime} & =\phi p^{\prime}+ \\
& \frac{1}{R e_{\Sigma}}\left[\left(1+\phi^{2}\right) w^{\prime \prime}+\left(\frac{1}{\phi}+2 \phi\right) w^{\prime}\right] .
\end{aligned}
$$

In the above, $R e_{x}$ is a Reynolds number based on the local value of $z$ and the primes denote differentiation by $\phi$. Since the Reynolds number has a $z$-dependence, the conical assumption does not truly hold. Conical self-similarity may therefore hold only in a "local" sense.

As with Burgers' vortex, Reynolds number dependence may be seen most easily by scaling the variables. Introducing the scaled variables

$$
\begin{aligned}
\tilde{u} & =u \sqrt{R e_{x}} \\
\tilde{v} & =v \\
\tilde{w} & =w \\
\tilde{p} & =p \\
\tilde{\phi} & =\phi \sqrt{R e_{x}}
\end{aligned}
$$

and dropping the terms that are higher-order in $R e_{z}$, the continuity equation becomes

$$
-\tilde{\phi}\left(\tilde{w}-\frac{\tilde{u}}{\bar{\phi}}\right)^{\prime}+2 \frac{\tilde{u}}{\tilde{\phi}}=0,
$$

the r-momentum equation becomes

$$
\tilde{p}=\frac{\tilde{v}^{2}}{\tilde{\phi}}
$$

the $\theta$-momentum equation becomes

$$
\tilde{v}^{\prime \prime}+\left[\frac{1}{\tilde{\phi}}+\tilde{\phi}\left(\tilde{w}-\frac{\tilde{u}}{\tilde{\phi}}\right)\right] \tilde{v}^{\prime}-\left[\frac{1}{\tilde{\phi}^{2}}+\frac{\tilde{u}}{\tilde{\phi}}\right] \tilde{v}=0
$$

and the $z$-momentum equation becomes

$$
\tilde{w}^{\prime \prime}+\left[\frac{1}{\tilde{\phi}}+\tilde{\phi}\left(\tilde{w}-\frac{\tilde{u}}{\tilde{\phi}}\right)\right] \tilde{w}^{\prime}+\tilde{v}^{2}=0,
$$

where the primes now denote differentiation with respect to $\tilde{\phi}$.

As written, there are no parameters in these equations - they enter only through the boundary conditions. The equations require one boundary condition each for $p$ and $u$, and two each for $v$ and $w$. The boundary conditions chosen for the velocities are

$$
\begin{array}{rlll}
\tilde{u}=0 & \text { at } & \tilde{\phi}=0 \\
\tilde{v}=0 & \text { at } & \tilde{\phi}=0 \\
\tilde{w}^{\prime}=0 & \text { at } & \tilde{\phi}=0 \\
\tilde{w}=1 & \text { at } & \tilde{\phi}=\tilde{\phi}_{\max } \\
\tilde{\phi} \tilde{v} \sim \tilde{\Gamma} & \text { at } & \tilde{\phi}=\tilde{\phi}_{\max }
\end{array}
$$

where the last boundary condition is implemented as

$$
\tilde{v}^{\prime}=-\tilde{\Gamma} / \tilde{\phi}^{2} \text { at } \tilde{\phi}=\tilde{\phi}_{\max }
$$

This system of equations for the velocities is rewritten as a set of five first-order equations and solved using centered differences and a Newton procedure. The equation for the pressure is then integrated independently, to give the static pressure coefficient $C_{p}$. The total pressure coefficient,

$$
C_{h}=\frac{p_{0}-p_{\infty}}{\frac{1}{2} \rho W^{2}}
$$

is calculated from $C_{p}$ and the velocities.

There are two parameters in the boundary conditions; $\tilde{\Gamma}$ and $\tilde{\phi}_{\text {max }}$. Solutions may be characterized by a single parameter, however. This parameter, $v_{e}$, is the maximum circumferential velocity of the vortex. Plots of the velocities and pressures for three different values of $v_{e}$ are shown in Figures 3-6. As the edge circumferential velocity increases, the axial velocity surplus in the core increases and the static and total pressures in the core decrease. As can also be seen, the gradients of circumferential velocity and total pressure are much more localized about the axis of the vortex than are the gradients of axial velocity and static pressure.

The scaled circulation $\tilde{\Gamma}$ is related to the physical circulation $\Gamma$ by the relation

$$
\tilde{\Gamma} \sim 2 \pi \Gamma \sqrt{R e_{z}} .
$$




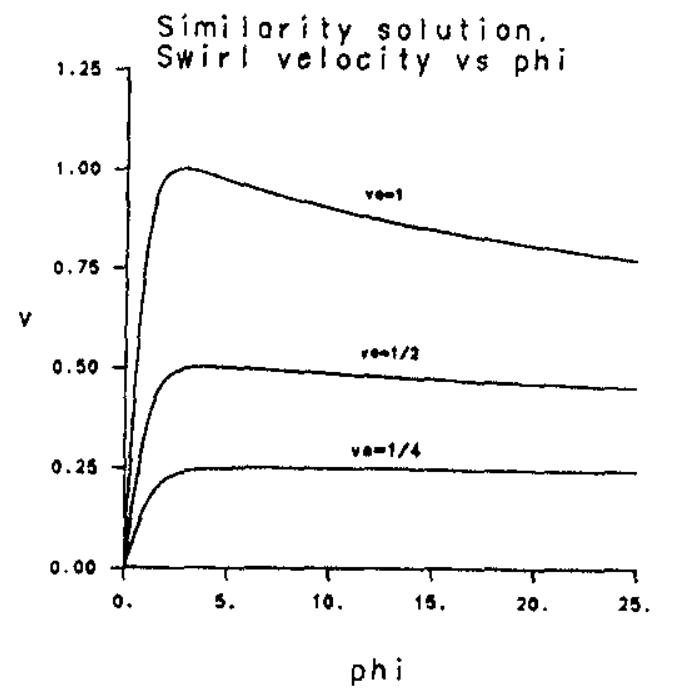

Figure 3: Conical vortex $-\tilde{v}$ vs $\tilde{\phi}$

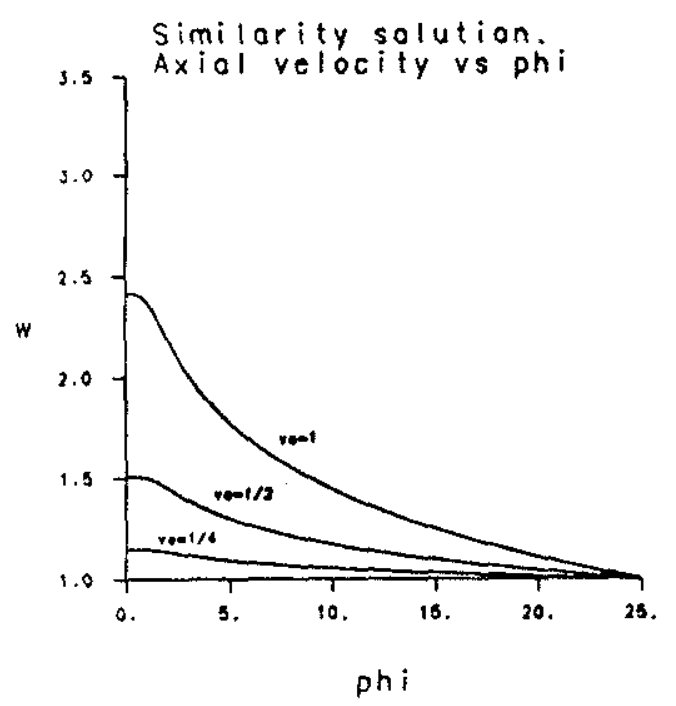

Figure 4: Conical vortex $-\tilde{w}$ vs $\tilde{\phi}$

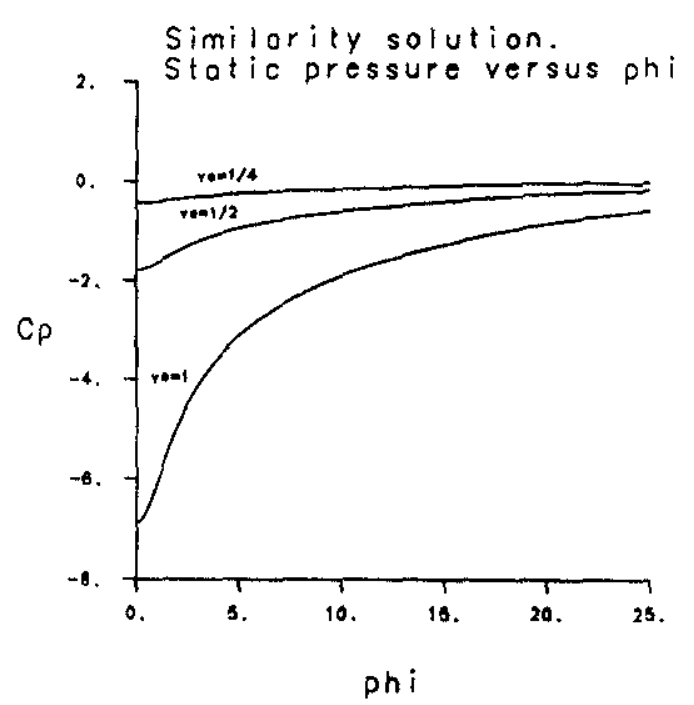

Figure 5: Conical vortex $-C_{p}$ vs $\tilde{\phi}$

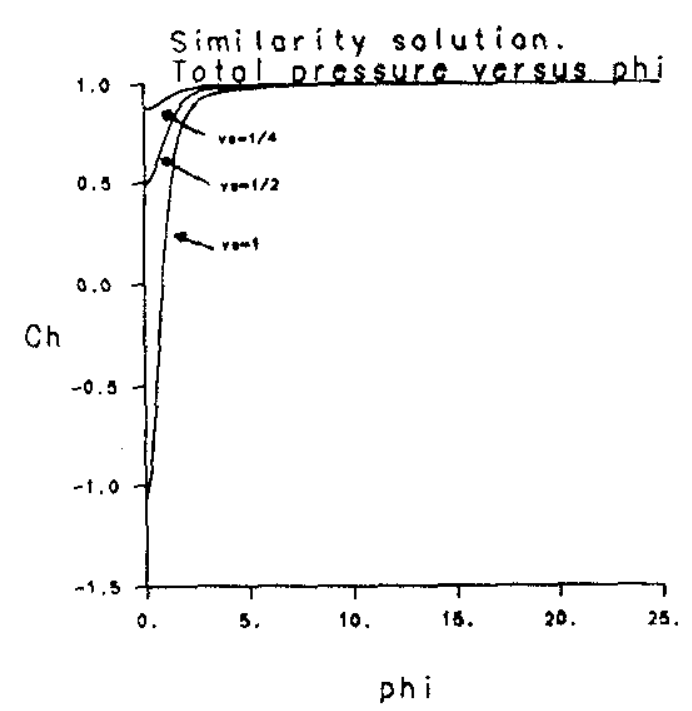

Figure 6: Conical vortex $-C_{h}$ vs $\tilde{\phi}$ 


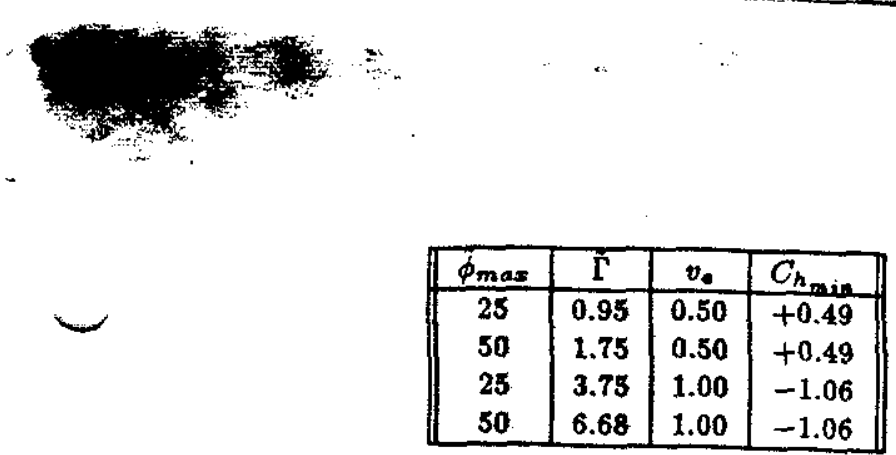

Table 1: vo and $C_{h_{\min }}$ as functions of $\dot{\Gamma}$ and $\dot{\phi}_{\text {max }}$

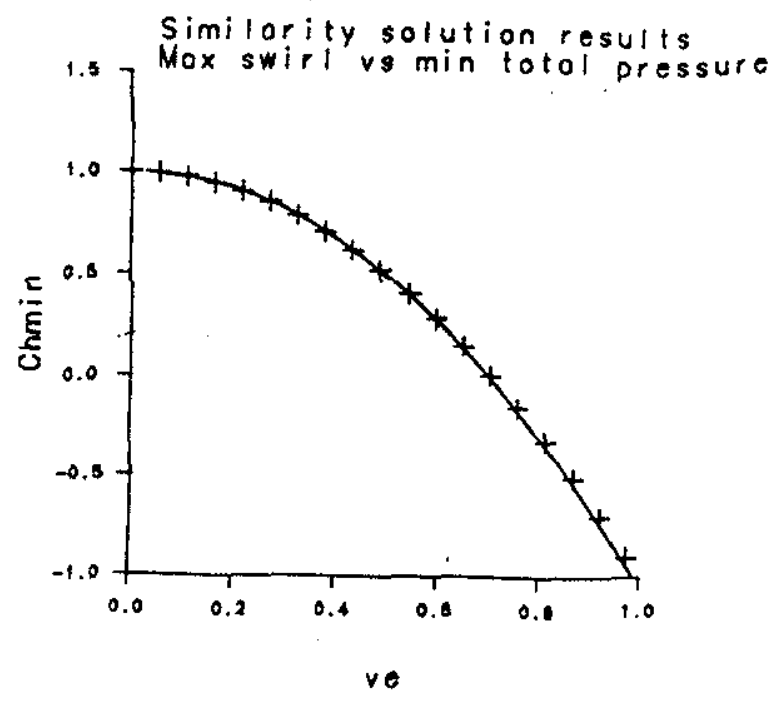

Fignre 7: Conical vortex $-C_{h_{\min }}$ vs $v_{\mathrm{o}}$

The size of the vortex scales with $1 / \sqrt{R e_{2}}$. Thus, as was the case with Burgers' vortex, it is the circumferential velocity $v_{e}$ that sets the level of the total pressure lose in the core. That this is true may be seen in Table 1 , where the maximam circumferential velocity $v_{a}$ and the minimum total pressure are tabulated for several values of $\tilde{\phi}_{\text {max }}$ and $\tilde{\Gamma}$. The variation of $C_{h_{\text {min }}}$ with $v_{s}$ is shown in Figure 7 . The solid line is the result of the conical model; the symobls are the curve

$$
C_{h_{\text {min }}}=1-2 v_{e}^{2} .
$$

This is the functional relationship that occurs for a very simple core model [16] in which:

1. radial velocity is negligible;

2. circumferential velocity is uniform and vanishes on the axis;

3. changes in static pressure and axial velocity cancel in the total pressure relation.

Thus, as with Buxgers' vortex, the total pressure loss is proportional to the square of the edge circumferential velocity of the vortex.

\section{Comparison of Results}

A comparison of the conical model with experimental measurements of the core of a leading-edge vortex for a delta wing is shown in Figures 8-11. The experiment was carried out by Earnshaw [1]. In the figures, the solid line represents the conical model and the symbols represent the experimental data. The spatial coordinate is normalized by the semi-span of the wing. The calculation was carried out so as to match the Reynolds number and maximum swirl velocity of the experiment.

The large gradient in circumferential velocity in the core is modeled well, however the rate of decay of circumferential velocity far from the axis is underpredicted. The axial velocity surplus in the core is overpredicted, as is the suction peak in the vortex. The level of total pressure loss on the axis is matched with experiment; hovever the width of the loosy region is drastically underpredicted.

The shortcomings of the model could be attributed to three features of the experiment which are not included in the model: too low a Reynolds number, lack of axisymmetry due to the presence of the wing, or turbulence. The Reynolds number in the experiment was on the order of one million, so that the high-Reynolds number assumption should hoid. The traverwes through the measured vortex showed a certain amonnt of aymmetry, however not enough to invalidate the model. The real culprit appears to be turbulence. Since the measured vortex shows a more difinse lossy region, there must be a diffusive process in the experiment that is not modeled in the similarity solution. Matching the solution to the experiment at a higher Reynolds number improved the comparion for the total pressure loss distribution, but degrade' the results for the static pressure and swirl velocity.

Another effect that must be considered is experimental error. The total pressure loss was meanured with a five-hole probe that was locally aligned with the flow. The measured losay region is only two or three probe-widths in extent, however. In addition, the actual magnitude of the total pressure lose is small in comparison with the free-stream pressure. The total pressure lose coefficient, $C_{h}$, may be related to the incremental total pressure loss, giving

$$
1-\frac{p_{0}}{p_{0_{\infty}}}=\frac{\gamma M_{\infty}^{2}}{2+\gamma M_{\infty}^{2}}\left[1-C_{h}\right] .
$$

Thus for a core loss $C_{h_{m} \text { in }}=-1.0$, the total pressure loss is only three percent of the freestream total pressure, which could be dificult to measure accurately.

A comparison of the new conical core model with the matched asymptotic expansion of Hall [2] is shown in Figares 12-15. Here the agreement is very good. The circumferential velocity and the static presenre are matched extremely well. The axial velocity surplus is less in the conical model, and the total pressure loss in the core is greater.

The close comparison of the conical similarity solution and Hall's matched asymptotic expansion is due to marked similarities in the two derivations. Hall's model is composed of a viscous, non-conical inner solntion matched to an inviscid, conical outer solution. The inner equations bear a resemblance to the conical model presented here. Since Hall is matching to conical outer conditions, it is not surprising that his solution closely resembles the conical similarity solution. 
Similority sofutions. $v e=1.00$

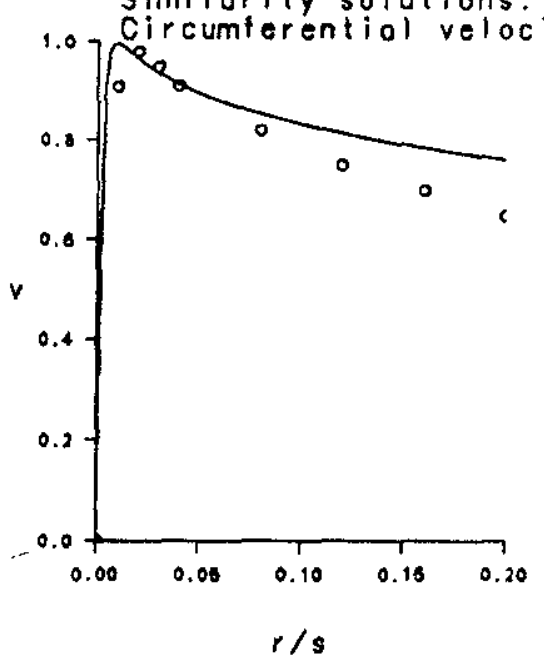

Figure 8: Comparison with Earnshaw - $v$ vs $\mathrm{r} / \mathrm{s}$

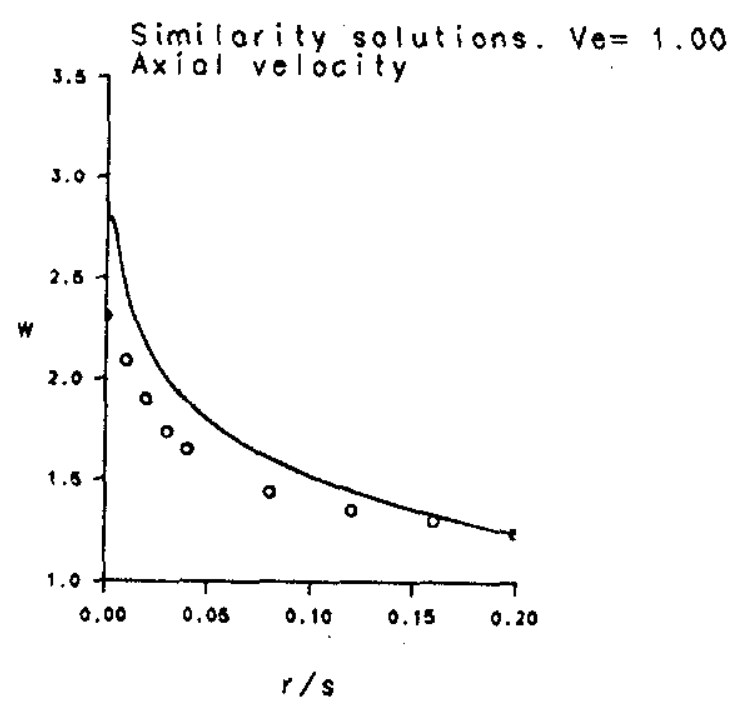

Figure 9: Comparison with Earnshaw - $w$ vs $r / s$

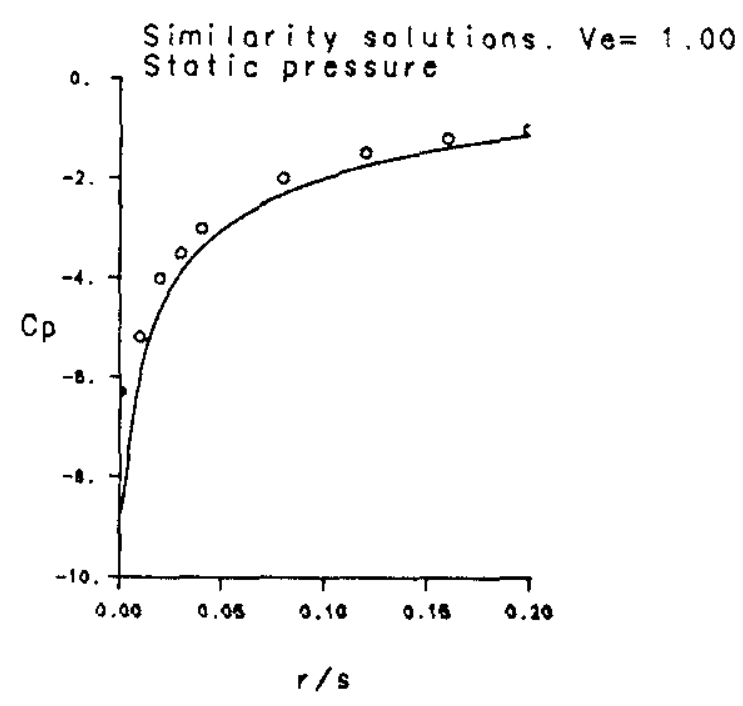

Figure 10: Comparison with Earnshaw $-C_{\mathrm{p}} \mathrm{vs} \mathrm{r} / \mathrm{s}$

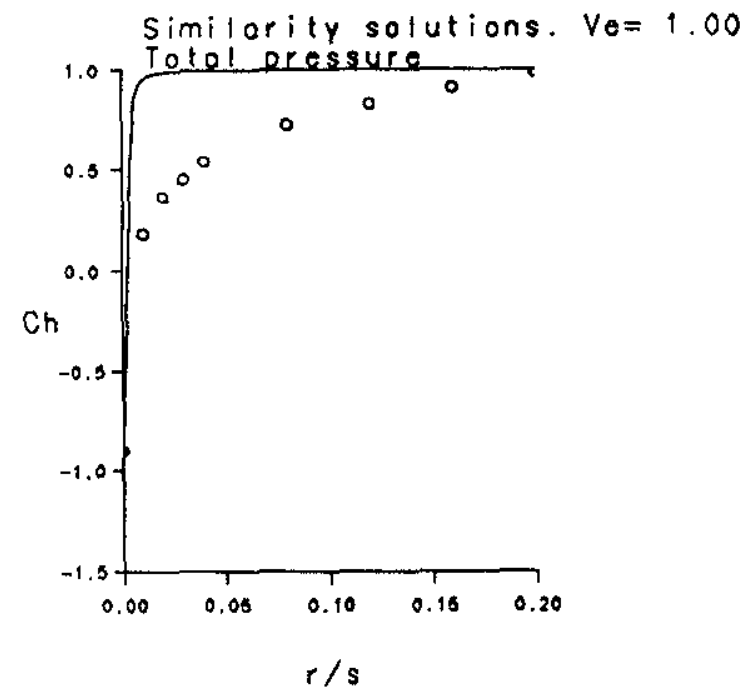

Figure 11: Comparison with Earnshaw - $C_{h}$ vs $r / s$ 


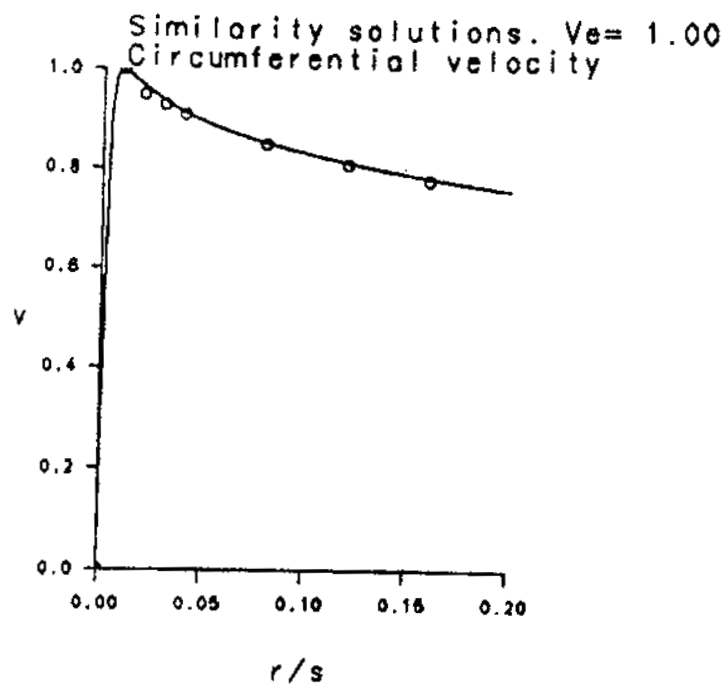

Figure 12: Comparison with Ball - v vs $\mathrm{r} / \mathrm{s}$

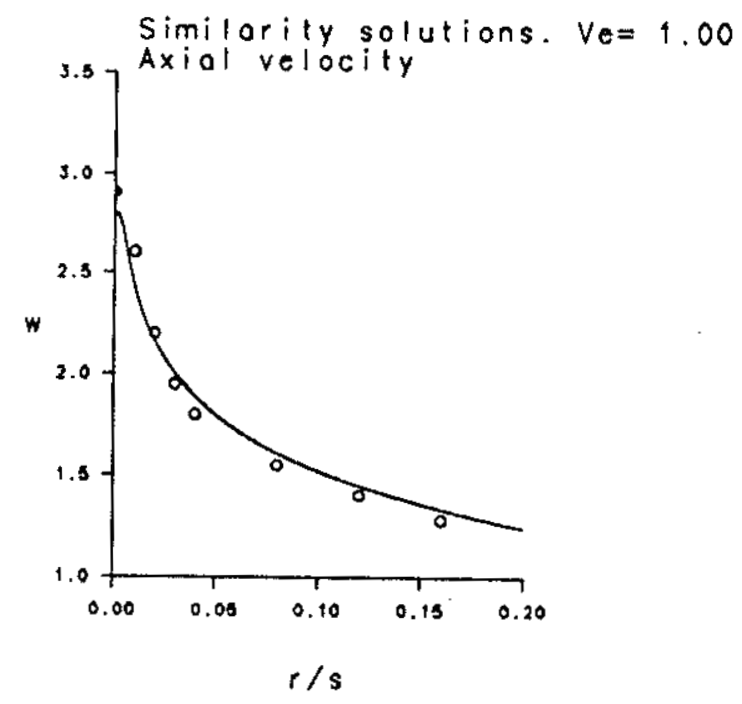

Figure 13: Comparison with Ball $-w$ vs $r / s$

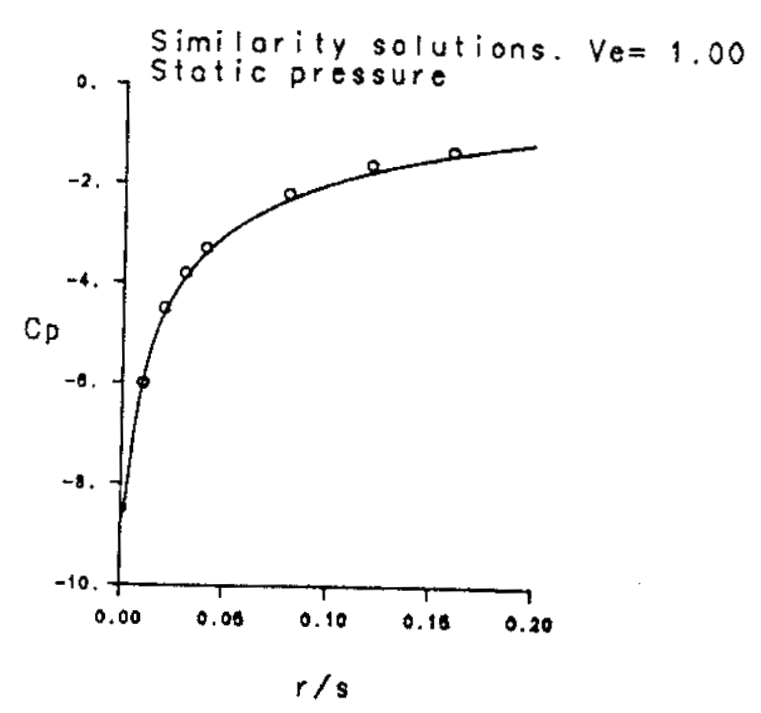

Figure 14: Comparison with Hall $-C_{p}$ vs $r / s$

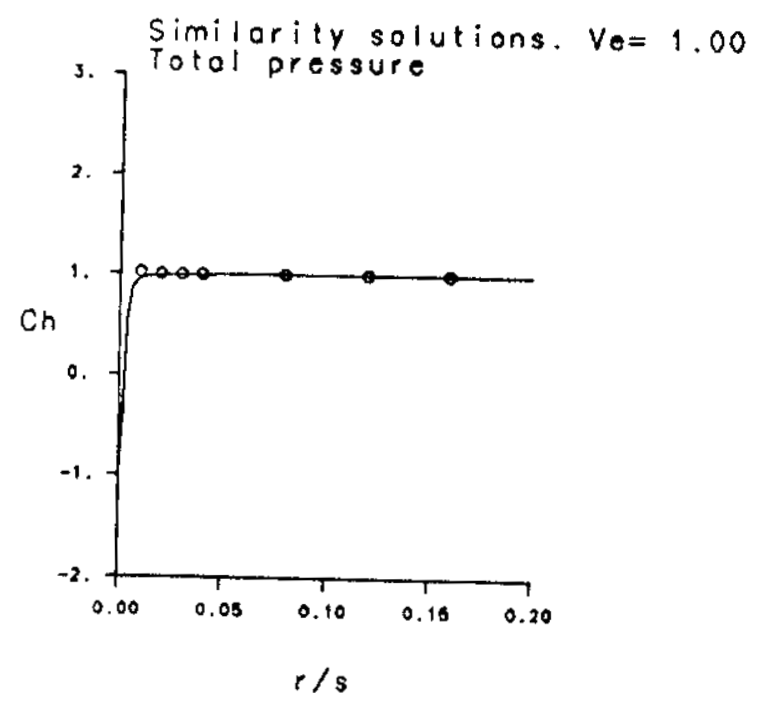

Figure 15: Comparison with Hall $-C_{h}$ vs $r / s$ 


\section{Discussion}

The model as it stands could help to give some ingight into prediction of the onset of vortex bursting. Experiments show that the magnitude of the core total pressnre loss greatly affects the occurence of bursting [17]. Several theoretical model relate the onset of bursting to a "critical swirl angle" [18]. The model developed in this paper shows a direct correlation between core total pressure lose and $v_{*}$, the edge swirl velocity of the vortex. Since the core total pressure lose and edge swirl velocity predicted by the model compare well with experiment, the onset of bursting should be predicted well by the model. Since the model is conical, it cannot model burating. It could, however, be used as an upstream condition for a vortex in a pressure gradient. Grabowski and Berger [19], for instance, carried out a numerical study of vortex bursting by assuming an upotream state for the vortex and solving the axisymmetric incompressible Navier-Stokes equations for the bursting of this vortex. Theix upatream state was an a priori function of the radius. Using a more physically justifiable upstream state could lead to more meaningful results. The core model presented here could fill that role.

Another advantage of the model is the generality of its derivation. Similarity equations for a compressible viscous core model can be derived by a process similar to the one demonstrated above. This has been done, yielding the set of ordinary differential equations below. They consist of the continuity equation

$$
-\tilde{\phi}\left[\tilde{\rho}\left(\tilde{w}-\frac{\tilde{u}}{\tilde{\phi}}\right)\right]^{\prime}+2 \frac{\bar{\rho} \tilde{u}}{\tilde{\phi}}=0,
$$

the $r$-momentum equation

$$
\frac{\gamma-1}{\gamma}(\tilde{\rho} \tilde{T})^{\prime}=\frac{\tilde{\rho} \tilde{v}^{2}}{\tilde{\phi}},
$$

the $\theta$-momentum equation

$$
\tilde{v}^{\prime \prime}+\left[\frac{1}{\tilde{\phi}}+\tilde{\rho} \tilde{\phi}\left(\tilde{w}-\frac{\tilde{u}}{\tilde{\phi}}\right)\right] \tilde{v}^{\prime}-\left[\frac{1}{\bar{\phi}^{2}}+\frac{\tilde{\rho} \tilde{u}}{\tilde{\phi}}\right] \tilde{v}=0
$$

the $z$-momentum equation

$$
\tilde{w}^{\prime \prime}+\left[\frac{1}{\tilde{\phi}}+\tilde{\rho} \tilde{\phi}\left(\tilde{w}-\frac{\tilde{u}}{\tilde{\phi}}\right)\right] \tilde{w}^{\prime}+\tilde{\rho} \tilde{v}^{2}=0,
$$

and the energy equation

$$
\begin{aligned}
&-\tilde{\rho} \tilde{\phi}\left(\tilde{w}-\frac{\tilde{\boldsymbol{v}}}{\tilde{\phi}}\right) \tilde{T}^{\prime}+\tilde{\rho} \tilde{w}^{2}\left(\tilde{w}-\frac{\tilde{u}}{\tilde{\phi}}\right)=\frac{1}{\operatorname{Pr}}\left(\tilde{T}^{\prime \prime}+\frac{\tilde{T}^{\prime}}{\tilde{\phi}}\right)+ \\
&\left(\tilde{w}^{\prime}\right)^{2}+\left(\tilde{v}^{\prime}-\frac{\tilde{v}}{\tilde{\phi}}\right)^{2}
\end{aligned}
$$

where the primes denote differentiation with respect to $\hat{\phi}$. In the above,

$$
\begin{aligned}
\tilde{T} & =\frac{T}{(\gamma-1) M_{\infty}^{2}} \\
\tilde{\rho} & =\rho
\end{aligned}
$$

and the other variables are scaled as before. The perfect gas law has been nsed to eliminate the pressure.

Again the equations of motion have been simplified to a set of coupled nonlinear ordinary differential equations. Solutions to this set of equations have not yet been obtained.

\section{Conclusions}

A model for an axisymmetric vortex in high Reynolds number flow has been presented. It is based on a new similarity solution to the Navier-Stokes equations. The model compares well with the previously existing model of Hall, due to the similarities in their derivations. The model compares well in a qualitative sense with experimental results. The measured vortex appeared more diffuse than the model, most probably owing to the effects of turbulence. The model is extendable to compressible flow, and shows promise in the prediction of the onset of vortex bursting.

\section{References}

[1] P. B. Earnshaw, An Experimental Investigation of the Structure of a Leading-Edge Vortex, Reports and Memoranda 3281, Aeronautical Research Council, 1961.

[2] M. G. Hall, "A Theory for the Core of a Leading-Edge Vortex," Journal of Fluid Mechanics, Vol. 11, 1961.

[3] R. R. Long, "A Vortex in as Infinite Viscous Fluid," Journal of Fluid Mechanics, Vot. 11, 1961.

[4] J. M. Luckring, "A Theory for the Core of a Three-Dimensional Leading-Edge Vortex," AIAA Paper 85-0108, 1985.

[5] S. N. Brown, "The Compressible Inviscid LeadingEdge Vortex," Journal of Fluid Mechanics, Vol. 22, 1965.

[6] K. G. Powell, Vortical Solutions of the Conical Euler Equations, ScD thesis, Masoachusetts Institute of Technology, 1987.

[7] K. G. Powell and E. M. Murman, "An Embedded Mesh Procedure for Leading-Edge Vortex Flows," In Proceedings of the Transonic Symposium, 1988, to appear.

[8] A. Rizzi, "Three-Dimensional Solutions to the Euler Equations with One Million Grid Points," AIAA Journal, Vol. 23, 1985.

[9] R. W. Newsome and J. L. Thomas, "Computation of Leading-Edge Vortex Flows," In Vortex Flow Aerodynamics - Volume I, NASA CP 2416, 1985.

[10] R. W. Newsome and O. A. Kandil, "Vortical Flow Aerodynamics - Physical Aspects and Numerical Simulation," AIAA Paper 83-1665, 1983.

[11] K. G. Powell, E. M. Murman, E. S. Perez, and J. R. Baron, "Total Pressure Loss in Vortical Solutions of the Conical Euler Equations," AIAA Journal, Vol. 25, 1987.

[12] E. M. Murman and K. G. Powell, "Comparison of Measured and Computed Pitot Pressures in a LeadingEdge Vortex from a Delta Wing," In Studies of VortexDominated Flows, Springer-Verlag, 1985, pp. 270-281. 
[13] P. M. Hartwich and C. H. Hsa, "An Implicit FluxDifference Splitting Scheme for Three-Dimensional, Incompressible Navier-Stokes Solutions to Leading-Edge Vortex Flow," In AIAA 4th Applied Aerodynamics Conference, 1986.

[14] D. P. Rizetta and J. S. Shang, "Numerical Simulation of Leading-Edge Vortex Flows," AIAA Paper 84-1544, 1984.

[15] J. M. Burgers, "A Mathematical Model Mlustrating the Theory of Tarbulence, ${ }^{n}$ In Advances in Applied Mechanics, Academic Press, 1948.

[16] J. H. B. Smith, Personal Communication, 1987.

[17] N. C. Lambourne and D. W. Bryer, The Bursting of Leading-Edge Vortices - Some Observations and Discussion of the Phenomenon, Reports and Memoranda 3282, Aeronantical Research Council, 1961.

[18] M. G. Hall, "Vortex Breakdown," Annual Reviev of Fluid Mechanics, 1972.

[19] W. J. Grabowski and S. A. Berger, "Solutions of the Navier-Stokes Equations for Vortex Breakdown," Journal of Fluid Mechanics, Vol. 75, 1976. 